\title{
LOS ARCTIIDAE Y SPHINGIDAE (LEPIDOPTERA: HETEROCERA) DEL SANTUARIO HISTÓRICO DE MACHU PICCHU, CUZCO, PERÚ: ESTUDIO PRELIMINAR.
}

\section{THE ARCTIIDAE AND SPHINGIDAE (LEPIDOPTERA: HETEROCERA) OF MACHU PICCHU HISTORICAL SANCTUARY, CUZCO, PERÚ: A PRELIMINARY SURVEY.}

\section{Juan Grados ${ }^{1}$}

\section{Resumen}

Se presenta una lista preliminar de 80 especies de Arctiidae y 17 de Sphingidae conocidas hasta el momento del Santuario Histórico de Machu Picchu, Cuzco, Perú, proporcionando datos de distribución altitudinal.

Palabras clave: Arctiidae, diversidad, Machu Picchu, Sphingidae, Perú.

\section{Summary}

A list of 80 species of Arctiidae and 17 of Sphingidae recorded until now at the Machu Picchu Historical Sanctuary, Cuzco, Perú, is presented herein, indicating their altitudinal distribution.

Keywords: Arctiidae, diversity, Machu Picchu, Sphingidae, Perú.

\section{Introducción}

E1 Santuario Histórico de Machu Picchu es el más importante atractivo turístico de nuestro país. La importancia histórica y arqueológica del lugar es conocida en todo el mundo, pero es recién a inicios de la década del 80 del siglo XX cuando sus recursos naturales de flora y fauna son reconocidos con relevancia al ser declarada el área como Santuario Histórico, dentro del Sistema de Áreas Naturales Protegidas por el Estado (SINANPE). La posibilidad de conjugar la conservación de un lugar histórico con la de la naturaleza hace del Santuario uno de los lugares más hermosos del Perú. Importantes contribuciones acerca de la parte histórica, así como estudios sobre geología, arqueología,

1 Museo Nacional de Historia Natural, Universidad Nacional Mayor de San Marcos, Apartado 14-034, Lima 14, Perú. E-mail: gradosjuan@hotmail.com arquitectura, flora y fauna se hallan en una obra editada por la Universidad San Antonio Abad del Cuzco (Chevarría, 1992).

Quizás uno de los primeros en colectar lepidópteros en áreas de lo que ahora es el Santuario Histórico de Machu Picchu haya sido Otto Garlepp (1864-1959) (Lamas et al., 1999), donde por error se cita al hermano de éste, Gustav Garlepp (1862-1907). Es así que he podido encontrar que existen cuatro registros de Ctenuchinae colectados por O. Garlepp con la localidad "Vilcanota" (3000 m), los mismos que fueron descritos por Hampson (1901) y Gaede (1926).

La expedición de la Universidad de Yale (EE. UU.) hacia el Perú, liderada por Hiram Bingham, tuvo como naturalista al químico Harry W. Foote, quien se encargaría de la colecta y preparación de la flora y fauna de la expedición. El 24 de julio de ese mismo año, 
día del descubrimiento de Machu Picchu, el valle del río Urubamba amaneció con una ligera llovizna, como cuenta el propio $\mathrm{H}$. Bingham. El Sr. Melchor Arteaga, quien conocía las ruinas y era guía de $\mathrm{H}$. Bingham, no tenía ninguna intención de salir a caminar a mostrar las ruinas que conocía. Luego de acceder por una considerable paga, ninguno de los otros acompañantes tenía esperanza de encontrar algo interesante en la parte alta, a donde se dirigirían en busca de las mencionadas ruinas. Como anécdota, podemos señalar que el naturalista H. W. Foote desecha la idea de acompañar a H. Bingham, aduciendo que había " ¡más mariposas cerca del río!", teniendo la certeza de colectar nuevas variedades para la ciencia $(\mathrm{H}$. Bingham, 1953; A. Bingham, 1989).

Los resultados de las colectas de H. W. Foote en la expedición de 1911 se dieron a conocer a través de varias publicaciones. En el caso de los Lepidoptera, Harrison Dyar (1913) del Museo Nacional de los Estados Unidos (USNM) publicó una lista de 242 especies de mariposas diurnas y nocturnas recolectadas en diferentes lugares del sur del Perú, incluyendo Machu Picchu. A este primer trabajo sobre lepidópteros, tenemos que agregar los trabajos sobre mariposas diurnas llevados a cabo por Hughes $(1958,1960,1962)$ y Lamas et al. (1999), registrándose en este último 252 especies. Últimamente se ha registrado a Brachygyna incae como nuevo género y especie (Lepidoptera: Psychidae) de mariposa nocturna para el Santuario Histórico de Machu Picchu (Davis, 2000).

\section{Material y métodos}

Las colectas de los Arctiidae y Sphingidae se llevaron a cabo entre el 17 y 29 de mayo de 1997 y el 20 de octubre y 03 de noviembre del 2001. Las colectas nocturnas se realizaron con una trampa de luz de mercurio de $250 \mathrm{~V}$ y en tres puntos fundamentales de muestreo: Río Alcamayo (1950 m; 1309/7231), Puente Ruinas (2050 m; 1310/7232) y Wiñaywayna (2675 $\mathrm{m} ; 1311 / 7232)$. Además se colectaron espe- cies de hábitos diurnos entre Aguas Calientes y Mandor (1800-1950 m; 1309-7233).

En la lista de los Arctiidae he añadido las tres especies registradas por Dyar (1913) que aún no he colectado: Virbia elisa Dyar, Virbia catama Dyar e Hypercompe peruviensis Hampson. Las dos primeras colectadas en la hacienda Huadquiña y la última en la antigua misión jesuita conocida como Santa Ana. Es casi seguro que las tres especies ocurren en las inmediaciones de la localidad del Santuario conocida como San Miguel. Además, incluyo las cuatro especies de Arctiidae colectadas por O. Garlepp en 1898, ya que al igual que la fauna de mariposas colectadas por él y que son típicas de Machu Picchu (Lamas et al., 1999), deben estar presentes también dentro de los límites del Santuario.

Las localidades para los Sphingidae son las mismas que para los Arctiidae capturados con trampa de luz, adicionando Aellopos titan (Cramer) que figura en la publicación de Dyar (1913), colectado en Huadquiña durante la expedición, además de existir un ejemplar en la colección del Museo Nacional de Historia Natural (MUSM), de la localidad de Calca, Río Urubamba.

Las muestras testigo se encuentran en el Museo Nacional de Historia Natural, Universidad Nacional Mayor de San Marcos (MUSM), Lima.

\section{Resultados y discusión}

\section{Arctiidae}

Las tablas 1 y 2 muestran dos columnas de ocurrencia de las especies indicando su altitud de colecta; la primera columna presenta la ocurrencia de las especies entre los 1800 2200 m (Alcamayo, Aguas Calientes, Puente Ruinas y Mandor) y la segunda la ocurrencia entre 2600-2700 m (Wiñaywayna).

La clasificación de la familia aún no es muy clara, existiendo algunas controversias con respecto al ordenamiento filogenético 
Tabla 1. Lista de los Arctiidae del S. H. Machu Picchu.

\section{Familia Arctiidae}

1800-2200 m

2600-2700 m

\section{Arctiinae}

1. Symphlebia similis (Rothschild, 1917)

2. Bertholdia specularis (Herrich-Schäffer, [1853])

3. Bertholdia crocea Schaus, 1910

4. Bertholdia grisescens Rothschild, 1909

5. Bertholdia flavidorsata Hampson, 1901

6. Hyperthaema ca. perflammans Hampson, 1916

7. Pachydota nervosa (R. Felder, 1874)

8. Pachydota striata (Dognin, 1893)

9. Ischnocampa nubilosa (Dognin, 1892)

10. Pelochyta arontes (Stoll, 1782)

11. Elysius atrata (R. Felder, 1874)

12. Elysius terraoides Rothschild, 1909

13. Opharus procroides Walker, 1855

14. Phaegoptera decrepidiodes (Rothschild, 1909)

15. Hemihyalea fulvizonata (Hampson, 1909)

16. Hemihyalea watkinsi (Rothschild, 1916)

17. Amastus coccinator Schaus, 1901

18. Amastus persimilis Hampson, 1901

19. Amastus ferrera Schaus, 1927

20. Amastus hyalina (Dognin, 1889)

21. Amastus sp. 1

22. Amastus sp. 2

23. Amastus sp. 3

24. Amastus sp. 4

25. Amastus sp. 5

26. Amastus sp. 6

27. Anaxita sannionis Butler, 1873

28. Halysidota intensa Rothschild, 1909

29. Lophocampa distincta (Rothschild, 1909)

30. Lophocampa modesta (Kirby, 1892)

31. Leucanopsis apicepunctata (Schaus, 1905)

32. Agaraea ockendeni Rothschild, 1909

33. Aemilia mincosa (Druce, 1906)

34. Aemilia rubriplaga Walker, 1855

35. Virbia elisca Dyar, 1913

36. Virbia catama Dyar, 1913

37. Hypercompe nemophila (Herrich-Schäffer, [1853])

38. Hypercompe peruviensis (Hampson, 1901)

39. Hypercompe cuninguda (Stoll, 1781)

40. Hypomolis ockendeni (Rothschild, 1910)

41. Hypomolis viridoides Toulgoët, 1982

42. Paracles sp.

43. Chloroda sp. n.

44. Utetheisa ornatrix ornatrix (Linnaeus, 1758)

$+$

$+$

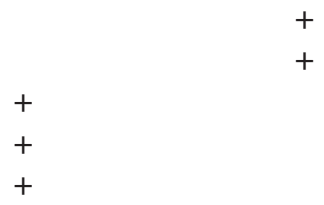


Pericopinae

45. Crocomela imperialis (Druce, 1885)

46. Crocomela flammifera (Warren, 1904)

47. Dysschema imitata (Druce, 1910)

48. Dysschema eurocilia staudingeri (Druce, 1910)

49. Dysschema hypoxantha ssp. $\mathrm{n}$

50. Dysschema sacrifica (Hübner, 1831)

\section{Ctenuchinae}

\section{Ctenuchini}

51. Telioneura rosada (Dognin, 1895)

52. Napata sanguicincta Hampson, 1901

53. Cyanopepla hurama (Butler, 1876)

54. Cyanopepla sanguicincta Gaede, 1926

55. Eucereon confinis (Herrich-Schäffer, 1855)

56. Eucereon myrtusa Druce, 1884

57. Eucereon davidi (Dognin, 1889)

58. Eucereon pseudocasca Rothschild, 1912

59. Eucereon nebulosum Dognin, 1889

60. Eucereon carabayana Schaus, 1905

61. Eucereon vestalis (Schaus, 1892)

62. Eucereon sp.n.

63. Eucereon arpi (L. Travassos, 1952)

64. Pionia oberthuri (Hampson, 1898)

65. Hyaleucerea morosa Schaus, 1910

Euchromiini

66. Sarosa notata (Butler, 1876)

67. Dasysphinx flavibasis Gaede, 1926

68. Andrenimorpha jordani (Zerny, 1912)

69. Mesothen petosiris (Druce, 1883)

70. Mesothen nomia Druce, 1900

71. Mesothen ockendeni Druce, 1905

72. Chrostosoma auge (Linnaeus, 1767)

73. Chrostosoma joavana (Schaus, 1924)

74. Rhynchopyga elongatus (Dognin, 1890)

75. Rhynchopyga xanthospila Hampson, 1898

76. Rhynchopyga bicolor (Dognin, 1902)

77. Macrocneme immanis Hampson, 1898

78. Phaio quadriguttata Dognin, 1909

79. Phaio coeruleonigra Schaus, 1905

80. Chrysocale corax Hampson, 1901
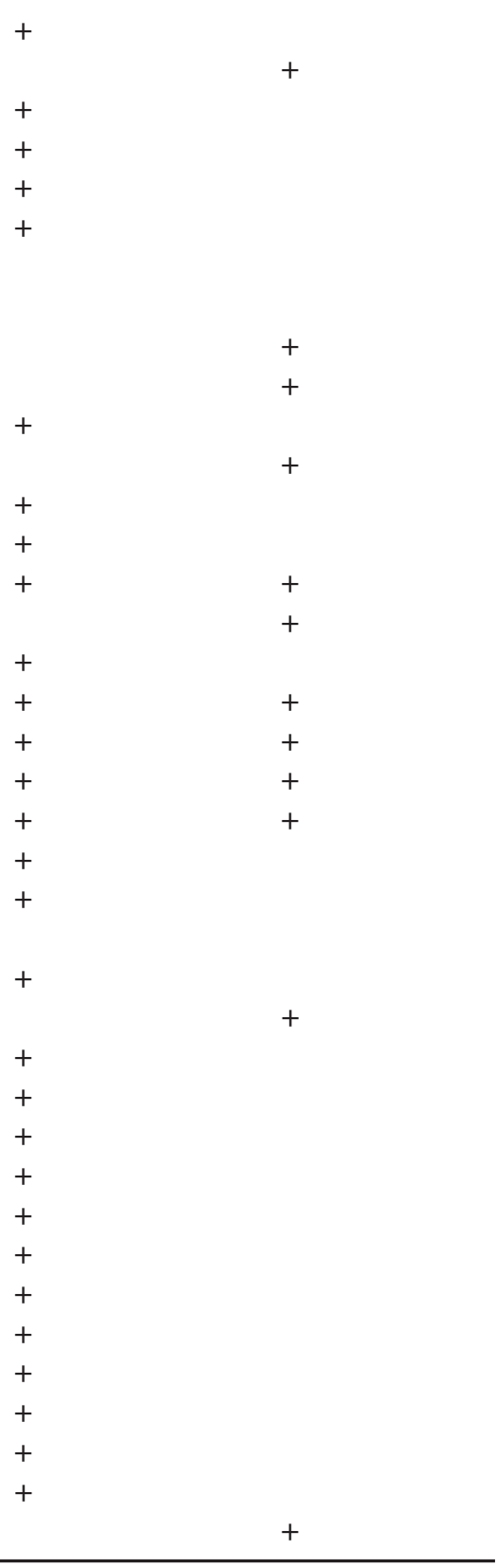
(Kitching \& Rawlins, 1999; Scoble, 1992; Weller et al., 1999). Tradicionalmente se han considerado cuatro subfamilias: Arctiinae, Lithosiinae, Ctenuchinae y Pericopinae, aunque Scoble (1992) propone que los Thyretinae, un pequeño grupo de aproximadamente 200 especies que ocurren fundamentalmente en África, son también Arctiidae, y Kitching \& Rawlins (1999) los consideran una tribu dentro de los Syntominae.

A fines del siglo pasado se propuso un ordenamiento filogenético de Arctiidae basado en el estudio de caracteres de adultos y larvas, proponiendo Weller et al. (1999), siguiendo a Kitching y Rawlins (1999) tres subfamilias: Lithosiinae, Syntominae y Arctiinae. El problema radica que en los aludidos trabajos, los análisis se han realizado con especies representativas de sólo algunos géneros, no especificando la localización de muchos otros, quedando la mayoría de los géneros en ubicación incierta. Mientras no se tenga criterios rigurosos de clasificación y ubicación determinada de ciertos taxones dentro de la clasificación propuesta, he optado por seguir en líneas generales el orden propuesto por Weller et al. (1999) con cierta flexibilidad, pues mantengo aún las subfamilias clásicas, considerando los Ctenuchinae con sus dos tribus (Grados, 1999a, 2001). El orden de los géneros de los Arctiinae y Pericopinae es siguiendo a Watson \& Goodger (1986) y el de los Ctenuchinae es según Draut (1916-1919).

La tabla 1 registra la ocurrencia de 80 especies de Arctiidae para el Santuario Histórico de Machu Picchu. La mayor diversidad encontrada se ubica en la parte baja (1800$2200 \mathrm{~m})$, con 63 especies $(79 \%)$, mientras en la alta se tiene 30 especies $(37,5 \%)$. La fauna compartida que se tiene hasta el momento es de 13 especies (16\%); es decir pocas especies tienen una distribución altitudinal amplia. Las altas montañas cubiertas por densa vegetación que encajonan el río Urubamba (conocido también como Vilcanota en ese sector) permiten dos diferentes tipos de fauna tan cercanos latitudinalmente, pero separados por un considerable gradiente altitudinal. De esta manera podemos encontrar fauna característica de la parte baja, colectada en Alcamayo y Puente Ruinas fundamentalmente. La zona con bosque más denso del Santuario, el sector de Mandor y sus montañas cubiertas de vegetación, es la que presumo debe albergar la mayor diversidad, fundamentalmente de Arctiidae y otras familias de mariposas nocturnas.

La zona de Wiñaywayna (2600-2700) registra por ahora una menor diversidad, teniendo en cuenta que el muestreo en la aludida zona fue tan sólo de dos días. El poco conocimiento de la fauna de las partes altas de los Andes peruanos se debe a la escasez de muestreos intensivos. Aún así, en los últimos años se han descrito varias especies nuevas para el Perú (Toulgoët, 1999, 2000a, b). Un ejemplo del escaso conocimiento lo tenemos en el género Amastus Walker, típico de zonas altas, del cual registro en este trabajo seis especies. Amén del pobre conocimiento de la fauna altoandina para el Neotrópico, el género consta de más de 130 especies, varias de ellas por describir, y que son en algunos casos bastante similares, lo que dificulta las determinaciones. Es necesario realizar más muestreos así como estudios morfológicos más detallados y análisis de los tipos.

Desde hace algunos años vengo realizando estudios taxonómicos y biogeográficos de los Arctiidae en diferentes lugares del Sudeste del Perú. De acuerdo a los datos que por el momento dispongo, la fauna de Arctiidae de Machu Picchu es bastante similar a la encontrada en el Valle de Cosñipata, también en el Cuzco.

\section{Sphingidae}

La tabla 2 muestra la ocurrencia de 17 especies de Sphingidae en el S. H. Machu Picchu. Los Sphingidae es un grupo de mariposas nocturnas cuyo conocimiento taxonómico es bien conocido, mas no así la distribución geográfica de las especies peruanas. La mayoría son de hábitos nocturnos, lle- 
gando a las trampas de luz con regularidad, pero algunas especies son crepusculares y su vuelo es de tan sólo algunos instantes alrededor de la luz y luego desaparecen (Kitching \& Cadiou 2000), por ello la rareza de algunas especies en colecciones y la importancia de colectar durante toda la noche.

Existen algunos trabajos sobre este grupo de lepidópteros en nuestro territorio, el realizado para la Zona Reservada Tambopata Candamo con 47 especies (Lamas, 1985), el inventario de la Cordillera del Cóndor, que registra 45 especies (Lamas, 1997), dos trabajos para la ciudad de Lima y alrededores con 18 especies (Moss, 1912; Lamas, 1981) y para la Zona Reservada de Tumbes se registró 21 especies (Grados, 1999b). Para otras zonas inventariadas de la Región Neotropical, ver Lamas (1985) y Grados (1999).

Una buena observación de una de las especies colectadas, Pseudosphix tetrio Linnaeus, fue registrada por Hughes (1960) en una de las visitas que realizó a Machu Picchu, señalando que era muy común, habiendo observado docenas de ellos acercándose a las luces. La lista presentada en este trabajo, siendo aún preliminar, creo será útil para un mejor conocimiento de la fauna de invertebrados del Santuario, ya que al igual que en el caso de los Arctiidae, no hay una lista elaborada anteriormente. En este caso podemos observar que los Sphingidae tienen una distribución altitudinal amplia, registrando ocho especies (47\%) para las dos zonas altitudinales de muestreo. De éstos, por registros de otras localidades del Perú y que constan en la colección MUSM, habría otras especies presentes en las dos zonas muestreadas, esperándose por ahora un total de 14 especies $(82 \%)$ compartidas entre las dos zonas de muestreo.

Tendremos un conocimiento más detallado de la fauna intrínseca de cada ecosistema particular que posee el Santuario, al realizar en el futuro más recolecciones en la parte de

Tabla 2. Lista de especies de Sphingidae en el Santuario Histórico de Machu Picchu.

\section{Familia Sphingidae}

1. Agrius cingulata (Fabricius, 1775)

2. Manduca lichenea (Burmeister, 1856)

3. Euryglotis aper (Walker, 1856)

4. Protambulyx euryalus Rothschild \& Jordan, 1903

5. Adhemarius gannascus gannascus (Stoll, 1790)

6. Adhemarius sexoculata Grote, 1865

7. Pseudosphinx tetrio (Linnaeus, 1771)

8. Erinnys alope alope (Drury, 1773)

9. Erinnys ello ello (Linnaeus, 1758)

10. Nyceryx hiposticta (R. Felder, [1874])

11. Perigonia stulta Herrich-Schäffer, [1854]

12. Aellopos titan (Cramer, 1777)

12. Xylophanes ceratomioides (Grote \& Robinson, 1867)

13. Xylophanes docilis (Butler, 1875)

14. Xylophanes pyrrhus (Rothschild \& Jordan, 1906)

15. Xylophanes crotonis (Walker, 1856)

16. Xylophanes nabuchondonosor Oberthür, 1904

17. Xylophanes resta Rothschild \& Jordan, 1903
$1800-2200 \mathrm{~m}$

$2600-2700 \mathrm{~m}$

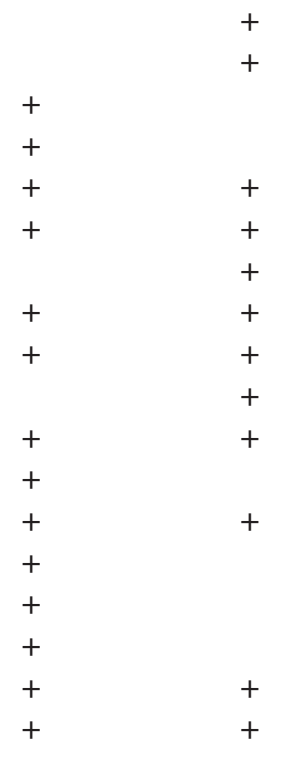


Puna del Camino Inca (Wayllabamba, Warmi Wañusca y Phuyupatamarca), la zona de San Miguel, que es la zona seca del Santuario,y por último, la zona aparentemente más diversa, Mandor y sus montañas cubiertas por densa vegetación.

\section{Agradecimientos}

Deseo agradecer a José Koechlin por su hospitalidad e interés en conocer la diversidad entomológica del Santuario Histórico de Machu Picchu. A INRENA y la Unidad de Gestión del Santuario proporcionaron los permisos de colecta. Un agradecimiento especial a G. Lamas, quien me invitó a realizar el estudio de Biodiversidad de los lepidópteros del Santuario Histórico de Machu Picchu.

\section{Literatura citada}

Bingham, A. M. 1989. Portrait of an explorer. Hiram Bingham, discoverer of Machu Picchu. Ames, Iowa State University Press.

Bingham, H. 1953. La ciudad perdida de los Incas. Historia de Machu Picchu y sus constructores. Editorial Zig-Zag, Santiago de Chile, 308 pp.

Chevarría, E. 1992. Machupicchu. Devenir Histórico y Cultural. Cuzco, UNSAAC, 250 pp.

Davis, D. 2000. Brachygyna incae, a new genus and species of Psychid from Peru with atypical larval biology. Tropical Lepidoptera, 10(2): 51-58 Draudt, M. 1916-1919. 3. Family: Syntomidae. In: Seitz A. (Ed.), Die GrossSchmetterlinge der Erde. Stuttgart, A Kernen. 6: 33-230.

Dyar, H. G. 1913. Results of the Yale Peruvian expedition of 1911. Lepidoptera. Proc. U.S. nat. Mus. 45 (2006): 627-649. Gaede, M. 1926. Amatiden des Berliner Zoologischen Museums. Dt. Ent. Z. 2: 113-136.

Grados, J. 1999a. Lista preliminar de los Ctenuchinae (Lepidoptera: Arctiidae) de la Zona Reservada Tambopata-Cándamo, Madre de Dios, Perú. Rev. per. Ent. 41: 9-14.

Grados, J. 1999b. Lista Preliminar de los Sphingidae y Saturniidae (Lepidoptera) de la Zona Reservada de Tumbes, Tumbes, Perú. Rev. per. Ent. 41: $15-18$.

Grados, J. 2001. Lista de los Ctenuchinae (Lepidoptera: Arctiidae) de la región del Bajo Urubamba, Cuzco, Perú. Rev. per. Ent. 42: 61-67.

Hampson, G. 1901. New species of Syntomidae and Arctiadae. Ann. Mag. nat. Hist. 7(8): 165186.
Hughes, R. A. 1958. Butterfly collecting in the high Andes of southern Peru, March 1956. Entomologist 91 (1136): 1-8, (1137): 33-37. - 1960. Tropical Migrants at Cuzco, Peru. Entomoligist 93:189-190. - 1962. Notes on the butterfly fauna of Machupicchu, South-east Peru. Entomologist 95(1186): 65-73.

Kitching, I. J. \& J.M. Cadiou. 2000. Hawkmoths of the World. An Annotated and Illustrated revisionary checklist (Lepidoptera: Sphingidae). The Natural History Museum, London \& Cornell University Press, Ithaca, USA, 226 pp.

Kitching, I. J. \& J. E. Rawlins. 1999. The Noctuoidea, pp. 355-401, In: Niels P. Kristensen (Ed.). Lepidoptera, Moths and Butterflies, Vol 1: Evolution, Systematics, and Biogeography, Walter de Gruyter, Berlin \& New York, 462 pp.

Lamas, G. 1981. Arthur Miles Moss (1873-1948) y los Sphingidae de Lima. Bol. Lima 14: 88-94.

Lamas, G. 1985. The Castniidae and Sphingidae (Lepidoptera) of the Tambopata Reserved Zone, Madre de Dios, Perú: A preliminary list. Rev. per. Ent. 27: 55-58.

Lamas, G. 1997. Lepidoptera of the Cordillera del Cóndor, pp. 90-98, 212-230,. In: Schulenberg, T. Awbrey K. (Eds.). The Cordillera del Cóndor Region of Ecuador and Peru: A biological assesment. RAP Working Papers 7: 1-231.

Lamas, G., J. Grados, y G. Valencia. 1999. Las mariposas de Machu Picchu, Cuzco, Perú: Un inventario preliminar (Lepidoptera: Rhopalocera). Rev. per. Ent. 41: 1-8.

Moss, A. M. 1912. On the Sphingidae of Peru. Trans. zool. Soc. London 20: 73-134.

Scoble, M. 1992. The Lepidoptera. Form, Function and Diversity. The Natural History Museum \& Oxford University Press, London, 404 pp.

Toulgoët, H. 1999. Description de nouvelles Arctiides néotropicales (Lepidoptera, Arctiidae, Arctiinae). Lambillionea 16(4): 357-360.

Toulgoët, H. 2000a. Description de nouvelles Arctiides d'Amerique du Sud (Lepidoptera Arctiidae Arctiinae). Lambillionea 17(2): 137-140.

Toulgoët, H. 2000b. Description d'une nouvelle Arctiide néotropicale (Lepidoptera Arctiidae Arctiinae). Lambillionea 17(2): 181-184.

Watson, A. \& D.T. Goodger . 1986. Catalogue of the Neotropical Tiger-moths. Occ. Pap. syst. Ent. (London) 1: 1-71.

Weller, S. J., N. L. Jacobson \& W. E. Conner. 1999. The evolution of chemical defenses and mating systems in tiger moths (Lepidoptera: Arctiidae). Biol. J. Linn. Soc. 68: 557-578. 